Revista de Psicología Vol. 33 (2), 2015 (ISSN 0254-9247)

\title{
Validación de una escala de estrés cotidiano en escolares chilenos
}

\author{
Yonatan José Encina Agurto y Myriam Viviana Ávila Muñoz ${ }^{2}$ \\ Universidad de Talca \& Fundación Educacional San Miguel Arcángel
}

\begin{abstract}
Se evaluaron las propiedades psicométricas de una escala de estrés cotidiano en el contexto escolar (EECE). Se estudió la estructura factorial y la consistencia interna en una muestra de niños y adolescentes chilenos de la Región del Maule $(n=734)$ con edades entre 10 y 17 años. La solución factorial de tres factores resultó simple y coherente respecto a las principales dimensiones del contexto escolar que pueden llegar a ser estresantes para los estudiantes: estrés académico, estrés relacional y ambiente escolar inadecuado. Los índices de consistencia interna fueron buenos tanto para la escala general como para los tres factores. Además se aprecian diferencias significativas en la percepción de estrés según el sexo (hombre/mujer) y ubicación geográfica (rural/urbano), que son coherentes con lo que señala la literatura. Palabras claves: estrés cotidiano, escolares, escuela, Escala Estrés.
\end{abstract}

\section{Validation of a scale on daily stress in school-age Chileans}

This study examines the psychometric properties of a scale of daily stress in the school context (EECE). The factorial structure and internal consistency were studied in a sample of Chilean children and adolescents from the Maule region $(n=734)$ between 10 to 17 years old. The factorial structure of three factors was simple and coherent with the main dimensions of the school context that can become stressful for students: academic stress, relationship stress and inadequate school climate. The internal consistency was good for both the overall scale as well as for the three factors. Also, significant differences were found in the perception of stress according to sex (male / female) and geographic location (rural / urban). These findings are consistent with the existing literature.

Keys words: Daily Stress, Students, School, Stress Scale.

1 Licenciado y magíster en Psicología. Dirección postal: Arauco, 794, Linares, Chile. Contacto: yencina@utalca.cl

2 Licenciada en Educación y magíster en Educación Especial y psicopedagogía. Dirección postal: Rio Yelcho 0880 Linares, Chile. Contacto: avila.myriam@gmail.com 
Si bien es posible apreciar que han aumentado los problemas de salud mental en niños y niñas (Marchesi, 2004) y que los problemas de psicopatología en la edad escolar son indicadores de alto riesgo para la salud adolescente y adulta (Florenzano \& Valdés, 2004), solo recientemente el sistema educativo reconoce formalmente el hecho de que en la escuela se dan procesos de desarrollo emocional que en sí mismos son indicadores de una educación de calidad. Prueba de ello es que el Ministerio de Educación de Chile (2014) en las últimas evaluaciones de calidad de las escuelas considera algunos indicadores socioemocionales, tales como autoestima, motivación y hábitos de vida saludable.

A pesar de la importancia de aquello, se puede decir que el desarrollo socioemocional ha sido un área infra-investigada en el contexto escolar, quedando en segundo plano respecto al desarrollo cognitivo (Berger et al., 2009). Esto es aún más significativo en términos del bienestar socioemocional de los escolares, pues como algunos autores señalan (Oyanedel, Alfaro, Varela \& Torres, 2014) realizada en Chile durante el alu00f1o 2012, en el marco de la Encuesta Internacional sobre Bienestar Subjetivo Infantil (ISCWeB la investigación respecto al bienestar (y podríamos agregar el malestar) subjetivo en niños y adolescentes en América Latina es particularmente escasa.

Uno de los indicadores que permiten introducirse al binomio bienestar/malestar subjetivo es el estrés cotidiano, es decir, aquellas frustrantes demandas y contrariedades que acarrea la interacción cotidiana con el medio, que son de alta frecuencia, baja intensidad y alta predictibilidad (Trianes et al., 2009). Lo relevante del estrés cotidiano, al menos en adolescentes, es que se asocia directamente con el desarrollo socioemocional de aquellos (Kiang \& Buchanan, 2014), por ejemplo como un predictor de ajuste emocional y conductual (Escobar, Fernández-Baena, Miranda, Trianes \& Cowie, 2011), y que puede llegar a tener impactos mayor en el bienestar de los sujetos que el producido 
por estresores vitales o crónicos (Seiffge-Krenke, 2000; Trianes et al., 2009). Esto por cuanto la mayoría de los eventos estresantes en la infancia y adolescencia son problemas que ocurren todos los días, como los conflictos en la escuela o peleas con los padres o los compañeros (Gelhaar et al., 2007; Seiffge-Krenke, 2000).

Existen distintos enfoques para acercarse al estudio del estrés en general (Escobar, Blanca, Fernández-baena \& Trianes, 2011; Vázquez, Crespo \& Ring, 2000) y sus subtipos (cotidiano, eventos vitales, agudo). Uno de los más fructíferos en el campo de la psicología es el transaccional, en cuanto se entiende al estrés como una "evaluación subjetiva sobre, primero, la existencia de un potencial estresor, y segundo, la inexistencia de habilidades o recursos para afrontarlo" (Trianes et al., 2009, p. 598).

En ese sentido, se pueden distinguir dos procesos cognitivos de valoración de la situación de estrés: por una parte la valoración primaria que refleja las percepciones sobre la naturaleza y el riesgo potencial (o la amenaza) de una situación; y por otra una valoración secundaria que refiere a la percepción de los recursos o habilidades para hacer frente a la situación propia de cada individuo (Govaerts \& Grégoire, 2004). Dentro de esta mirada cognitiva del proceso emocional, se asume por ende que un suceso es estresante en la medida que el sujeto la perciba o valore como tal (Escobar, Fernández-Baena, Miranda, Trianes \& Cowie, 2011; Morales, Trianes \& Miranda, 2012; Vázquez et al., 2000) Aquello facilita la aplicación de autoinformes para captar la experiencia emocional que un estresor despierta en las personas (Trianes et al., 2009).

\section{Estresores en distintos dominios durante la infancia y adolescencia: focalización el proceso educativo}

Hay un amplio consenso en la literatura que aborda el fenómeno del estrés en la infancia y la adolescencia sobre el hecho de que existen distintos dominios en los cuales se generan situaciones estresantes 
para los individuos, dado el período del desarrollo evolutivo en que se encuentran. Los principales que han sido descritos e investigados son: la familia, la escuela, los pares y en menor medida el contexto socioeconómico o comunitario (Escobar et al., 2011; Khamis, 2013; Kiang \& Buchanan, 2014; Morales et al., 2012; Trianes et al., 2009). Aunque muchos estresores de la infancia pueden llegar a ser considerados universales, por cuanto han sido encontrado en diversas muestras interculturales, algunos son específicos del contexto en el que se estudian (Khamis, 2013) por ejemplo los socioculturales, como lo muestra un estudio con estudiantes asioamericanos (Kiang \& Buchanan, 2014) u otro con nińos de la Franja de Gaza (Khamis, 2013), por lo que es valioso considerar dichas diferencias contextuales al momento de crear o utilizar técnicas e instrumentos de medición estandarizados.

Dentro del proceso educativo, a nivel de la escuela, es posible identificar "subdominios" específicos en los cuales se pueden generar situaciones potenciales de estrés para los estudiantes, siendo los más descritos en la literatura el estrés académico y el relativo a las relaciones interpersonales con pares y profesores. El estrés académico de naturaleza cotidiana en el contexto escolar tiene que ver principalmente con las situaciones y experiencias ligadas directamente al proceso de enseñanza aprendizaje, y existe abundante literatura al respecto tanto a nivel teórico (Putwain, 2007, 2009) como empírico. Por ejemplo, los eventos que han sido descritos como estresores son aplicación de pruebas estandarizadas y su dificultad (llamado también estrés a los exámenes); cantidad y dificultad de tareas en la escuela y para la casa (Hjern, Alfven \& Ostberg, 2008; Ilias \& Nor, 2013); dificultad para entender lo que se enseña (Kiang \& Buchanan, 2014); altas expectativas respecto al rendimiento escolar del estudiante, su familia (Escobar et al., 2011; Ilias \& Nor, 2013; Kiang \& Buchanan, 2014) y la escuela (Putwain, 2009); bajas calificaciones (Escobar et al., 2011; Trianes et al., 2009) y el abatimiento generado por el proceso de aprendizaje (Ilias \& Nor, 2013; Sun, Dunne, Hou \& Xu, 2013).

En cuanto a las situaciones estresantes de carácter relacional, dadas por las interacciones con pares y profesores en la escuela se han descrito 
como estresores cotidianos en la escuela las discusiones con compañeros y amigos en la escuela, así como el grado de demandas de aquellos (Kiang \& Buchanan, 2014); la ridiculización por parte de los compañeros, el trato injusto, y la falta de aceptación o rechazo (Escobar et al., 2011; Hjern et al., 2008; Morales \& Guerra, 2006; Trianes et al., 2009); además de los cambios abruptos de grupos de amigos (Kiang \& Buchanan, 2014).

Por su parte en la diada maestro-alumno se ha visto que es estresante tener problemas relacionales con los profesores (Escobar et al., 2011; Trianes et al., 2009) como por ejemplo un trato injusto o inadecuado por parte de los maestros (Hjern et al., 2008); además de experimentar distancia afectiva y falta de guía mostrada por los profesores, así como un estilo coercitivo de enseñanza (Meijer, 2007).

También se puede distinguir un tercer dominio que es fuente probable de situaciones de estrés cotidiano para los estudiantes, aunque es menos claro y descrito en las investigaciones, que puede ser llamado ambiente adecuado/inadecuado para el desarrollo, en cuanto al conjunto de situaciones en un nivel más general (de curso o escuela) que pueden llegar a ser más o menos amenazantes para los estudiantes. Desde esta línea algunos autores proponen (Martínez-Otero, 2012) que la excesiva burocratización de las organizaciones escolares (es decir, que ponen mucho énfasis en los resultados y los indicadores por sobre las relaciones interpersonales, se vuelven más individualistas, predomina un estilo verticalista y rígido de los directivos), genera espacios menos acogedores y por ende con mayor probabilidades de ser fuentes de estrés para los estudiantes. Incluso existe evidencia de que el contacto con la naturaleza del entorno residencial y del entorno escolar tiene un efecto amortiguador sobre el estrés de los niños (Corraliza \& Collado, 2011). Por otra parte a nivel del aula se ha visto que el tipo de ambiente de aprendizaje que genera el profesor tiene un efecto en el bienestar de los estudiantes, pues quienes generan una atmosfera colaborativa y orientada a la tarea obtienen mejores resultados académicos y bienestar con sus alumnos (Meijer, 2007). 


\section{Medición del estrés cotidiano en nińos y adolescentes}

Existen varias discusiones teóricas y metodológicas abiertas respecto a la medición del estrés, sobre todo en el contexto escolar y particularmente en lo referido al estrés académico, tales como una falta de precisión en la terminología utilizada confundiéndose a veces el estrés con la ansiedad o la preocupación; la poca claridad en el concepto mismo de estrés ya que a veces se utiliza para referirse a las propiedades de un estímulo (por ejemplo, una prueba) y en otros casos a la experiencia subjetiva de malestar, así como una tendencia a la utilización de métodos cuantitativos por sobre los cualitativos (Putwain, 2007).

A lo anterior, habría que agregar una dominancia de la investigación en población anglosajona, por lo que los modelos teóricos planteados, los instrumentos utilizados y los resultados obtenidos dan cuenta solo de ciertos contextos educativos, y es bien sabido que los distintos sistemas educacionales son capaces de crear distintas condiciones estructurales más o menos demandantes para sus actores, entre ellos los estudiantes, tal como lo muestran estudios en torno al fenómeno del estrés y afrontamiento en el caso de Europa (Gelhaar et al., 2007) y el de China (Chao, Wang \& Sung, 2012; Sun, Dunne, Hou \& Xu, 2011; Sun et al., 2013).

Más allá de estas discusiones lo claro es que en general se utilizan cuestionarios de autoreporte para medir la percepción de estrés, ya sea enfocándolo como estímulo (estresores) o como respuesta (experiencia y/o sintomatología ansiosa), a veces por medio de cuestionarios estandarizados (como los presentados en la Tabla 1) y otras veces creándose cuestionarios ad-hoc para el estudio con indicadores específicos (ver por ejemplo Galván \& Mcglennen, 2012; Geisthardt \& Munsch, 1996; Govaerts \& Grégoire, 2004; Hanson \& Chen, 2010; Hjern et al., 2008; Kiang \& Buchanan, 2014; Morales \& Guerra, 2006). 


\section{Tabla 1}

Algunos cuestionarios que miden estrés cotidiano en niños y adolescentes.

\begin{tabular}{|c|c|c|c|}
\hline Instrumento & Utilizado por & $\begin{array}{c}\text { Cantidad } \\
\text { Ítems }\end{array}$ & Dimensiones o factores \\
\hline $\begin{array}{l}\text { The Hassles } \\
\text { Scale for } \\
\text { Children } \\
\text { (HSC) }\end{array}$ & $\begin{array}{l}\text { Parfenoff \& Paul } \\
\text { (1989) }\end{array}$ & 49 & $\begin{array}{l}8 \text { dimensiones (autoestima } \\
\text { y bienestar, relaciones } \\
\text { familiares, escuela, salud } \\
\text { personal, entre otros) }\end{array}$ \\
\hline $\begin{array}{l}\text { The Educational } \\
\text { Stress Scale for } \\
\text { Adolescents } \\
\text { (ESSA) }\end{array}$ & $\begin{array}{l}\text { Sun et al., (2011); } \\
\text { Sun et al., (2013) }\end{array}$ & 16 & $\begin{array}{l}5 \text { dimensiones (presión por } \\
\text { el estudio, carga de trabajo, } \\
\text { entre otros) }\end{array}$ \\
\hline
\end{tabular}

The Adolescent Anda et al., (2000) $143 \quad 5$ dimensiones (estresores

Stress, Stressor, familiares, escolares, estresoand Coping res del mundo, entre otros

Measure

\begin{tabular}{|c|c|c|c|}
\hline $\begin{array}{l}\text { Adolescent } \\
\text { Hassles } \\
\text { Inventory (AHI) }\end{array}$ & Dumont (2000) & 59 & No especifíca \\
\hline $\begin{array}{l}\text { The Children's } \\
\text { stressors scale } \\
\text { (CSS) }\end{array}$ & Khamis (2013) & 31 & $\begin{array}{l}6 \text { dimensiones (estresores } \\
\text { familiares, económicos, } \\
\text { sociales, anomia, entre otros) }\end{array}$ \\
\hline $\begin{array}{l}\text { Inventario } \\
\text { Infantil de } \\
\text { Estresores } \\
\text { Cotidiano } \\
\text { (IECI ó IIEC) }\end{array}$ & $\begin{array}{l}\text { Escobar et al., (2011); } \\
\text { Morales et al., (2012); } \\
\text { Trianes et al., (2009); }\end{array}$ & 25 & $\begin{array}{l}3 \text { factores de segundo orden } \\
\text { (Problemas de salud y psi- } \\
\text { cosomáticos, escolares y } \\
\text { familiares) }\end{array}$ \\
\hline $\begin{array}{l}\text { Adolescent } \\
\text { Stress } \\
\text { Questionnaire } \\
\text { (ASQ) }\end{array}$ & $\begin{array}{l}\text { Escobar et al., } \\
\text { (2011) }\end{array}$ & 58 & $\begin{array}{l}7 \text { factores de primer orden } \\
\text { y uno de segundo (con- } \\
\text { flicto entre escuela y ocio, } \\
\text { asistencia escolar obligato- } \\
\text { ria, incertidumbre sobre el } \\
\text { futuro, entre otros) }\end{array}$ \\
\hline
\end{tabular}

*Fuente: elaboración propia con datos tomados de los estudios citados. 
Como se puede observar en la Tabla 1 existen distintos instrumentos que se pueden utilizar en la medición del estrés cotidiano en niños y adolescentes; sin embargo la mayoría de ellos fueron creados en el contexto de habla inglesa, y solo algunos han sido utilizados en población de habla hispana como por ejemplo el ASQ. Además varios son relativamente extensos (sobre 40 ítems). En español destaca el Inventario Infantil de Estresores Cotidianos de Trianes y colaboradores (2009). En el caso del contexto chileno no existen cuestionarios ni escalas estandarizadas que permitan medir el estrés cotidiano en la población escolar; existe un bajo número de investigaciones publicadas que aborden este fenómeno. Cabe destacar el estudio de Barra, Cerna, Kramm y Véliz (2006) quienes utilizaron el Perceived Stress Scale (Cohen, Kamarck \& Mermelstein, 1983). Sin embargo es una medida global que indaga que tan impredecible, incontrolable y sobrecargada se ha experimentado la vida durante el último mes, por lo que no permite identificar aspectos concretos del ambiente que puedan ser considerados estresantes; además no se reporta el proceso de adaptación y validación en el contexto nacional de dicho instrumento.

Por otra parte, todos los instrumentos que abordan la experiencia del estrés de manera general considerando las dimensiones familiares, de salud, personales, escolares, etc., son un aporte en el estudio de este fenómeno, y algunos han sido desarrollados para el contexto escolar por ejemplo el ESSA. Sin embargo, no existen cuestionarios estandarizados y publicados en espańol y en inglés que permitan medir de manera confiable y válida el fenómeno del estrés cotidiano en el contexto particular de la escuela en población infantil y adolescente, más allá del estrés académico; considerando los aspectos relacionales y de un ambiente para el desarrollo, además del ámbito académico.

Conforme a lo expuesto, en este trabajo se dan a conocer las principales propiedades psicométricas de la Escala de Estrés Cotidiano Escolar (EECE), que se propone como una herramienta que permite medir los principales factores y variables que se dan en el marco del proceso escolar y que son potencialmente una fuente de estrés para los estudiantes. 
De esta manera, este instrumento podrá ser utilizado en investigación básica y aplicada que permita evaluar posibles estresores de carácter cotidiano que se dan en el contexto escolar, y su impacto en el bienestar/malestar de los estudiantes así como su relación con otras variables relevantes.

\section{Método}

\section{Participantes}

Fueron encuestados 734 estudiantes de la Región del Maule (Chile) durante el segundo semestre del año 2013 en sus respectivos colegios. De ellos el $46.19 \%$ fueron mujeres y el $53.81 \%$ fueron varones. La edad media fue 12.75 años, con una desviación estándar de 1.23 ańos, y el rango fue de 10 a 17 años.

\section{Medidas e instrumentos de medición}

El estrés cotidiano en el contexto escolar fue medido por la EECE. El diseño general de este instrumento estuvo basado en lo que se ha llamado la valoración primaria dentro del enfoque transaccional, por ello primero se indaga la presencia de un estresor en la escuela y luego la valoración que hace el niño o adolescente respecto al hecho potencialmente estresante. Por ejemplo, se pregunta si ha sufrido burlas en la sala o en el patio del colegio, y luego se le pregunta qué tanto le molesta, asusta o incomoda (en escala Likert de 1 a 4) el sufrir discriminación en la sala o en el patio del colegio. Entendiendo que a mayor grado de molestia, incomodidad o miedo le genere el hecho mayor será su potencial como estresor para el estudiante.

Originalmente se redactaron 25 ítems (asumiendo que es un número razonable para aplicación con niños y adolescentes) organizados en 3 dimensiones: estresores relacionales, académicos y ambiente inadecuado para el desarrollo socioemocional. 
En primer lugar, se procedió a una revisión de los ítems por jueces expertos del área de la educación básica (4), educación diferencial (1) y de psicología (1), todos con grado de magister al menos en sus respectivas áreas. Luego se realizó una aplicación piloto el ańo 2012 con 180 estudiantes de educación básica (séptimo y octavo) y primero de media de la ciudad de Talca; de los cuales $60 \%$ fueron varones y $40 \%$ mujeres. En todo este proceso se procedió a eliminar 7 ítems que eran confusos y no aportaban significativamente a la consistencia interna de la escala, dificultando la interpretación y carga factorial luego de realizar un análisis exploratorio y de confiabilidad. Estos ítems eliminados estaban ligados con la disponibilidad y uso de drogas en la escuela, así como la experimentación de delitos violentos en la escuela o alrededor de ella. Por ello, la escala cuenta con 18 ítems.

\section{Procedimiento}

La aplicación de los instrumentos se realizó en las propias escuelas y en la sala de clases de cada grupo, obteniendo previamente la autorización de los directores. A los niños y adolescentes se les explicaron los objetivos del estudio y se les motivó a contestar con honestidad y seriedad los cuestionarios.

El procesamiento y análisis de datos se realizó con los programas SPSS versión 20, Factor versión 9.2 y AMOS versión 22. En primer lugar se realizaron análisis descriptivos a nivel de ítems, factores y escala completa. Luego se evaluó la confiabilidad de la escala por medio de del coeficiente $\alpha$ de Cronbach, antes de realizar un estudio de validez por medio de análisis factorial exploratorio usando el método de extracción de mínimos cuadrados no ponderados (Unweighted Least Squares o MLS). Para simplificar el modelo factorial se utilizó la rotación oblicua promin, dado que supone que todos los elementos de un cuestionario multidimensional en el plano psicosocial son medidas puras de un único rasgo es más bien un planteamiento poco realista (Ferrando \& Lorenzo-Seva, 2000). 


\section{Resultados}

En primer lugar se revisó la presencia de casos atípicos multivariados a través de la prueba de distancia de Mahalanobis $(p<.001)$ y no se identificaron casos que estuviesen fuera de los valores de criterio. Luego se verificó el supuesto de normalidad de los ítems para evaluar la pertinencia del análisis factorial exploratorio, mediante el cálculo del tamaño de la asimetria (skewness) y curtosis (Miles \& Shevlin, 2001). En este caso los valores de los ítems estuvieron bajo los parámetros establecidos $( \pm 1,5)$ tal como se puede apreciar en la tabla 2 .

\section{Tabla 2}

Media, desviación estándar, asimetría y curtosis de la distribución de los items de la EECE

\begin{tabular}{lcccc}
\hline Ítem & $M$ & $D E$ & Asimetría & Curtosis \\
\hline Ítem 1 & 2.13 & .95 & .53 & -.59 \\
Ítem 2 & 2.39 & 1.13 & .15 & -1.36 \\
Ítem 3 & 2.44 & 1.15 & .08 & -1.43 \\
Ítem 4 & 2.32 & 1.20 & .22 & -1.51 \\
Ítem 5 & 2.36 & 1.18 & .15 & -1.48 \\
Ítem 6 & 2.19 & .99 & .38 & -.92 \\
Ítem 7 & 2.05 & 1.14 & .62 & -1.09 \\
Ítem 8 & 2.31 & 1.31 & .23 & -1.46 \\
Ítem 9 & 2.08 & .98 & .52 & -.77 \\
Ítem 10 & 2.19 & .91 & .42 & -.58 \\
Ítem 11 & 2.28 & .92 & .30 & -.70 \\
Ítem 12 & 2.43 & 1.00 & .16 & -1.03 \\
Ítem 13 & 2.08 & 1.10 & .57 & -1.03 \\
Ítem 14 & 2.10 & 1.11 & .53 & -1.11 \\
Ítem 15 & 2.11 & 1.12 & .51 & -1.16 \\
Ítem 16 & 2.18 & 1.09 & .40 & -1.16 \\
Ítem 17 & 2.46 & 1.16 & .00 & -1.46 \\
Ítem 18 & 2.16 & 1.12 & .39 & -1.26 \\
\hline
\end{tabular}




\section{Análisis de datos}

Para el análisis de la estructura factorial se utilizó el método de los mínimos cuadrados no ponderados basado en una matriz de correlaciones policóricas, dada la naturaleza ordinal de los datos (Antino, Gil-Rodriguez, Martí, Barrasa \& Borzillo, 2014; Quintard et al., 2011). El modelo presenta buenos índices adecuación muestral, así, la prueba de Kaiser-Meyer-Olkin (KMO), fue de .89 y la prueba de esfericidad de Bartlett fue significativa $=6640.6 p=.000$. Además se evaluó la simplicidad del modelo por medio del índice de Bentler (1977) (llamado índice S) y la sencillez de carga (Lorenzo-Seva, 2003) (índice LS) cuyos valores fueron .99 y .70 respectivamente; ello indica que cada ítem está relacionado principalmente a una sola dimensión general, y que la solución general muestra una alta simplicidad factorial.

Para determinar el número de factores se recurrió a la conjugación de los criterios basados en al análisis paralelo (Horn, 1965) y la regla Kaiser-Gutman de autovalores mayores a 1, así como la coherencia y simplicidad teórica. De esta forma, se determinó que existen tres dimensiones subyacentes en los datos, por lo que la escala general puede considerarse tridimensional. La varianza total explicada por los factores fue de 11.61 es decir el $64.56 \%$ de aquella. Además la escala completa presenta un buen índice de consistencia interna $\alpha=.90$. Para facilitar la interpretación de las cargas factoriales se procedió a realizar rotación oblicua (Promin) dado que ofrece soluciones más precisas y reproducibles en factores correlacionados. Sin embargo igualmente se probó la solución factorial con rotación ortogonal Varimax, encontrándose una interpretación semejante. Tal como se observa en la Tabla 3, todos los reactivos tienen cargas aceptables factoriales aceptables siendo las más bajas las de los ítems 1, 7 y 8 . Además todos cargan en un solo factor.

Conforme a estos resultados se puede apreciar que los ítems se agrupan según el modelo propuesto originalmente en las dimensiones propuestas. En base a aquello se procedió a nombrar cada uno de los factores, siendo el factor 1 denominado "estrés académico" (ítems 9 al 12) 
y se refiere al grado de incomodidad o molestia que le provocan al estudiante (en mayor o menor medida) los estresores ligados a aspectos académicos en el contexto escolar. Por ejemplo: altos niveles de exigencia académica manifestada por los profesores, dificultad de tareas y pruebas escolares, cantidad de tareas, etc. Los niveles de consistencia interna son buenos $\alpha=.88$.

Por su parte el factor 2 se denominó "estrés de violencia relacional" (ítems 1 al 8) y se refiere al grado de incomodidad o molestia que le provocan al estudiante (en mayor o menor medida) los estresores ligados a violencia de tipo relacional en el contexto escolar. Por ejemplo: ver peleas en la escuela, ser víctima de robos, burla o discriminación en la escuela, ser aislado por los pares, etc. Para esta escala, los niveles de consistencia interna también son buenos $\alpha=.80$.

Finalmente el factor 3 se nombró "estrés ambiental" (ítems 13 al 18) se refiere al grado de incomodidad o molestia que le provocan al estudiante los estresores ligados a un ambiente inadecuado para su desarrollo integral en el contexto escolar. Por ejemplo: despreocupación y falta de disponibilidad de los profesores hacia los estudiantes, falta de actividades extracurriculares para todos los estudiantes, falta de motivación y reconocimiento de los profesores hacia los estudiantes, etc. Los niveles de consistencia interna son buenos Alfa de Cronbach .86.

\section{Diferencias según sexo y zona geográfica de residencia}

Finalmente, se evaluó el comportamiento del instrumento a través de las comparaciones de los puntajes totales (suma simple) observados a nivel de los factores y de la escala completa, con relación a dos variables demográficas: sexo (hombre-mujer) y ubicación geográfica (rural-urbano). Las comparaciones de grupos se realizaron a través del estadístico $U$ de Mann Withney, dado que las variables no cumplieron los supuestos para aplicar pruebas paramétricas como la prueba $t$ de comparación de medias. 
Validación de una escala de estrés cotidiano en escolares chilenos / Encina Agurto y Ávila Muñoz

\section{Tabla 3}

Matriz de estructura factorial de la EECE. Se omiten los reactivos que tuvieron un peso factorial igual o menor a.4

\begin{tabular}{|c|c|c|c|c|}
\hline Reactivos & $\mathrm{F} 1$ & $\mathrm{~F} 2$ & F3 & \multirow{5}{*}{$r_{\text {item-total }}$} \\
\hline Varianza explicada & 7.78 & 2.03 & 1.80 & \\
\hline$\%$ varianza explicada & 43.23 & 11.28 & 10.05 & \\
\hline $\mathrm{M}$ & 18.18 & 8.97 & 13.10 & \\
\hline$\overline{\mathrm{DE}}$ & 6.73 & 3.01 & 5.20 & \\
\hline Ítem 1 & & .45 & & .38 \\
\hline Ítem 2 & & .86 & & .61 \\
\hline Ítem 3 & & .90 & & .63 \\
\hline Ítem 4 & & .93 & & .67 \\
\hline Ítem 5 & & .90 & & .65 \\
\hline Ítem 6 & & .48 & & .56 \\
\hline Ítem 7 & & .48 & & .60 \\
\hline Ítem 8 & & .67 & & .65 \\
\hline Ítem 9 & .63 & & & .39 \\
\hline Ítem 10 & .92 & & & .42 \\
\hline Ítem 11 & .78 & & & .42 \\
\hline Ítem 12 & .66 & & & .40 \\
\hline Ítem 13 & & & .88 & .60 \\
\hline Ítem 14 & & & .85 & .55 \\
\hline Ítem 15 & & & .89 & .58 \\
\hline Ítem 16 & & & .71 & .56 \\
\hline Ítem 17 & & & .55 & .63 \\
\hline Ítem 18 & & & .69 & .59 \\
\hline
\end{tabular}


Tal como se puede observar en la tabla 4 tanto el sexo como la zona geográfica tienen una relación con la percepción de estrés que experimentan los escolares, donde las mujeres tienden a percibir mayor nivel de estrés que los hombres tanto a nivel general $(U=55819$, $p=.000)$, como a nivel relacional $(U=57397.5, p=.001)$, el estrés académico $(U=60375, p=.021)$ y el ambiente inadecuado de desarrollo $(U=58748.5, p=.004)$.

\section{Tabla 4}

Media y Desviación Estándar de la escala general y de cada factor, según sexo y zona geográfica.

\begin{tabular}{|c|c|c|c|c|c|c|c|c|}
\hline \multirow[t]{2}{*}{ Factor } & \multicolumn{2}{|c|}{$\begin{array}{l}\text { Varones } \\
N=395\end{array}$} & \multicolumn{2}{|c|}{$\begin{array}{l}\text { Mujeres } \\
N=339\end{array}$} & \multicolumn{2}{|c|}{$\begin{array}{c}\text { Urbano } \\
N=636\end{array}$} & \multicolumn{2}{|c|}{$\begin{array}{c}\text { Rural } \\
N=98\end{array}$} \\
\hline & $M$ & $D E$ & $M$ & $D E$ & $M$ & $D E$ & $M$ & $D E$ \\
\hline Escala Total & 38.60 & 11.41 & 42.21 & 12.64 & 40.80 & 11.98 & 36.82 & 12.53 \\
\hline $\begin{array}{l}\text { Estrés } \\
\text { Relacional }\end{array}$ & 17.38 & 6.42 & 19.12 & 6.97 & 18.45 & 6.70 & 16.46 & 6.70 \\
\hline $\begin{array}{l}\text { Estrés } \\
\text { Académico }\end{array}$ & 8.69 & 2.92 & 9.31 & 3.09 & 9.10 & 3.01 & 8.18 & 2.98 \\
\hline $\begin{array}{l}\text { Estrés } \\
\text { Ambiental }\end{array}$ & 12.53 & 4.86 & 13.77 & 5.51 & 13.25 & 5.14 & 12.17 & 5.50 \\
\hline
\end{tabular}

Por su parte los escolares que residen en zonas urbanas tienden a percibir mayor nivel de estrés que aquellos escolares que residen y estudian en un contexto rural, tanto a nivel general $(U=25111$, $p=.002)$, como a nivel relacional $(U=25525,5, p=.004)$, el estrés académico $(U=25662.5, p=.005)$ y el ambiente inadecuado de desarrollo $(U=27033.5, p=.034)$.

\section{Discusión}

En este estudio se procuró establecer las principales propiedades psicométricas de una escala que permita medir de manera confiable 
y válida el estrés cotidiano percibido en el contexto escolar por estudiantes. En términos generales los resultados indican que la EECE es un instrumento adecuado para valorar el estrés cotidiano en niños y nińas chilenos de entre 10 y 17 años tanto de contextos rurales como urbanos. Además, se destaca su eficiencia, pues es una escala consistente y relativamente corta en relación con otras que existen y que miden aspectos similares (ver Tabla 1). Se espera que su uso no solo sea posible con escolares chilenos, sino que se pueda ampliar a niños de habla hispana, tomando en consideraciones los aspectos contextuales de cada sistema educativo.

Respecto a la estructura factorial, los resultados fueron consistentes con las tres dimensiones propuestas respecto a ámbitos potencialmente estresantes del contexto escolar: el académico, las relaciones interpersonales y el ambiente escolar en general. Estos resultados son importantes dado que amplían el horizonte de fenómenos que son potencialmente fuente de estrés para los escolares, dado que la literatura en general se ha centrado en estudiar principalmente el estrés académico (Escobar et al., 2011; Hjern et al., 2008; Ilias \& Nor, 2013; Kiang \& Buchanan, 2014; Putwain, 2009; Trianes et al., 2009) o el relacional (Escobar et al., 2011; Hjern et al., 2008; Kiang \& Buchanan, 2014; Morales \& Guerra, 2006; Trianes et al., 2009); considerándolos además de manera parcelada.

En ese sentido, esta escala permite obtener indicadores parciales pero también totales de la experiencia de estrés cotidiano en los escolares, corroborado por los indicadores de consistencia interna de cada factor y de la escala en su conjunto, a pesar de la pequeña cantidad de ítems que la conforman.

Por otra parte, se pudo comprobar la capacidad discriminativa de la escala en relación a distintos grupos poblacionales. Así, se pudo observar resultados consistentes con la literatura respecto al tema del estrés en relación al sexo de los niños, niñas y adolescentes, pues estudios previos han mostrado como las mujeres tienden a reportar mayores niveles de estrés percibido en relación a los hombres (Escobar, Trianes, Fernández-Baena \& Miranda, 2010; Ministerio de Salud de Chile, 2011), 
tanto a nivel de las relaciones interpersonales como en las valoraciones que hacen de las exigencias académicas (Kiang \& Buchanan, 2014).

En ese sentido las niñas reportan mayor número de eventos negativos que los niños en sus vidas y, en algunos casos, se consideran en mayor riesgo que aquellos. Por ejemplo existe evidencia de que las nińas otorgan mayor importancia a las experiencias estresantes, mientras que los niños se perciben a sí mismos con más recursos para hacer frente a dichas experiencias (Govaerts \& Grégoire, 2004).

Por otra parte es interesante el constatar que los niños y niñas que viven y estudian en un contexto rural tienden a reportar menores niveles de estrés cotidiano en el contexto escolar. Si bien esta variable ha recibido menos atención en la literatura respecto a la salud mental infantil, existen estudios con población general en contexto rural donde aún no existen datos concluyentes que indiquen si los agricultores y familias de agricultores experimentan mayores tasas de problemas de salud mental en comparación con la comunidad no-agrícola; por lo que se requiere mayor investigación (Fraser et al., 2005).

Algunas posibles explicaciones en cuanto a esta diferencia entre escolares rurales y urbanos pueden estar relacionadas con lo que indican algunos estudios, en cuanto a que la naturaleza puede tener un efecto moderador del estrés infantil (Wells \& Evans, 2003), ya que quienes viven en un entorno rural tienen un contacto más cercano y estrecho con la naturaleza. En ese sentido, se ha visto que aquellos niños que tienen la posibilidad de disfrutar un mayor contacto con áreas verdes mostrarán menos estrés que aquellos que no tenga la posibilidad de pasar tiempo en contacto con el mundo natural (Corraliza \& Collado, 2011).

Los mecanismos explicativos podrían ir al menos por dos vías (Wells \& Evans, 2003): los niños que crecen en contextos rurales pueden recibir mayor apoyo social, pues crecen y se desarrollan interactuando cercanamente con otros niños en el medio natural sin muchas restricciones. La otra vía de explicación seńala que el contacto con el medio natural ayuda a restaurar la capacidad de enfocar la atención en las personas, ya que la naturaleza nos llama la atención sin esfuerzo (efecto de fascinación) lo que permite a los mecanismos inhibitorios que subyacen 
en el proceso atencional descansar; mientras que al mismo tiempo, la sensación de estar lejos de las preocupaciones cotidianas permite una sensación de relajación mental.

Respecto a líneas de investigación futura, se plantea la necesidad de seguir probando el funcionamiento psicométrico de la escala en más contextos escolares, tanto a nivel nacional como internacional (habla hispana), así como la evaluación de otras propiedades psicométricas como la validez concurrente (ya sea con otros instrumentos similares, o bien complementado el autoreporte con la información de otros informantes como profesores o padres). Además es importante probar la capacidad predictiva de la EECE respecto a otros indicadores de bienestar/malestar subjetivo en escolares, así como variables ligadas con el rendimiento académico.

\section{Referencias}

Anda, D. De, Baroni, S., Boskin, L., Buchwald, L., Morgan, J., Ow, J. \& Weiss, R. (2000). Stress, stressors and coping among high school students. Children and Youth Services Review, 22(6), 441-463.

Antino, M., Gil-Rodriguez, F., Martí, M., Barrasa, A. \& Borzillo, S. (2014). Desarrollo y validación de una versión española del Team Climate Inventory: un análisis de invariancia factorial. Anales de Psicología, 30(2), 597-607.

Barra, E., Cerna, R., Kramm, D. \& Véliz, V. (2006). Problemas de Salud, Estrés, Afrontamiento, Depresión y Apoyo Social en Adolescentes. Terapia Psicológica, 24(1), 55-61.

Bentler, P. M. (1977). Factor simplicity index and transformations. Psychometrika, 42(2), 277-295.

Berger, C., Milicic, N., Alcalay, L., Torretti, A., Arab, M. \& Justiniano, B. (2009). Bienestar socio-emocional en contextos escolares: la percepción de estudiantes chilenos. Estudios Sobre Educación, $17,21-43$. 
Chao, T.-Y., Wang, Y.-L. \& Sung, Y.-T. (2012). Development of Scale of Examination Stress and Test Anxiety (SESTA) and the Examination Stress in Taiwan. Poster presentado en la 120th American Psychological Association Annual Convention.

Cohen, S., Kamarck, T. \& Mermelstein, R. (1983). A global measure of perceived stress. Journal of Health and Social Behavior, 24(4), 385-396.

Corraliza, J. \& Collado, S. (2011). La naturaleza cercana como moderadora del estrés infantil. Psicothema, 23(2003), 221-226.

Dumont, M. (2000). Experience du stress a l'adolescence. Journal International de Psychologie, 35(5), 194-206.

Escobar, M., Blanca, M. J., Fernández-Baena, F. J. \& Trianes, M. V. (2011). Adaptación española de la escala de manifestaciones de estrés del Student Stress Inventory (SSI-SM). Psicothema, 23(3), 475-485.

Escobar, M., Fernández-Baena, F. J., Miranda, J., Trianes, M. V. \& Cowie, H. (2011). Low peer acceptance and emotional / behavioural maladjustment in schoolchildren: Effects of daily stress, coping and sex. Anales de Psicologia, 27(2), 412-417.

Escobar, M., Trianes, M. V., Fernández-Baena, F. J. \& Miranda, J. (2010). Relaciones entre Aceptación Sociométrica Escolar e Inadaptación Socioemocional, Estrés Cotidiano y Afrontamiento. Revista Latinoamericana de Psicología, 42(3), 469-479.

Ferrando, P. \& Lorenzo-Seva, U. (2000). Unrestricted versus restricted factor analysis of multidimensional test items: Some aspects of the problem and some suggestions. Psicológica: Revista de Metodologia y Psicología Experimental, 21(2), 301-323.

Florenzano, R. \& Valdés, M. (2004). El adolescente y sus conductas de riesgo. Santiago de Chile: Ediciones UC.

Fraser, C., Smith, K., Judd, F., Humphreys, J., Fragar, L. \& Henderson, A. (2005). Farming and Mental Health Problems and Mental Illness. International Journal of Social Psychiatry, 51(4), 340-349. 
Galván, A. \& Mcglennen, K. M. (2012). Daily Stress Increases Risky Decision-Making in Adolescents: A Preliminary Study. Developmental Psychobiology, 4, 433-440.

Geisthardt, C. \& Munsch, J. (1996). Coping with school stress: a comparison of adolescents with and without learning disabilities. Journal of Learning Disabilities, 29, 287-296.

Gelhaar, T., Seiffge-krenke, I., Borge, A., Cicognani, E., Cunha, M., Loncaric, D. \& Metzke, C. W. (2007). Adolescent coping with everyday stressors: A seven-nation study of youth from central, eastern, southern, and northern Europe. European Journal of Developmental Psychology, 4(2), 129-156.

Govaerts, S. \& Grégoire, J. (2004). Stressful academic situations: study on appraisal variables in adolescence. Revue Européenne de Psychologie appliquéeEuropean Review of Applied Psychology, 54(4), 261-271.

Hanson, M. D., \& Chen, E. (2010). Daily Stress, Cortisol, and Sleep: The Moderating Role of Childhood Psychosocial Environments. Health Psychology, 29(4), 394-402.

Hjern, A., Alfven, G. \& Ostberg, V. (2008). School stressors, psychological complaints and psychosomatic pain. Acta Paediatrica, 97(1), 112-117.

Horn, J. L. (1965). A rationale and test for the number of factors in factor analysis. Psychometrika, 30(2), 179-185.

Ilias, K. \& Nor, M. (2013). Stress and Satisfaction: Students at the Ipoh Teachers' Training Institute. European Social Sciences Research Journal, 1(2), 169-175.

Khamis, V. (2013). Stressors , family environment and coping styles as predictors of educational and psychosocial adjustment in Palestinian children. Educational Studies, 39(4), 371-384.

Kiang, L. \& Buchanan, C. (2014). Daily Stress and Emotional WellBeing Among Asian American Adolescents: Same-Day, Lagged, and Chronic Associations. Developmental Psychology, 50(2), 611-621. 
Lorenzo-Seva, U. (2003). A factor simplicity index. Psychometrika, 68(1), 49-60.

Marchesi, Á. (2004). Qué será de nosotros, los malos alumnos. Madrid: Alianza.

Martínez-Otero, V. (2012). El estrés en la infancia: estudio de una muestra de escolares de la zona sur de Madrid capital. Revista Iberoamericana de Educación, 59(2).

Meijer, J. (2007). Correlates of student stress in secondary education. Educational Research, 49(1), 21-35.

Miles, J. \& Shevlin, M. (2001). Applying regression and correlation: A guide for students and researchers. London: SAGE Publications.

Ministerio de Educación de Chile. (2014). Otros Indicadores de Calidad Educativa. Santiago de Chile: MINEDUC.

Ministerio de Salud de Chile. (2011). Encuesta nacional de salud. Chile 2009-2010. Santiago de Chile: MINSAL.

Morales, F., Trianes, M. \& Miranda, J. (2012). Diferencias por sexo y edad en afrontamiento infantil del estrés cotidiano. Electronic Journal of Research in Educational Psychology, 10(26), 95-110.

Morales, J. R. \& Guerra, N. G. (2006). Effects of Multiple Context and Cumulative Stress on Urban Children's Adjustment in Elementary School. Child Development, 77(4), 907-923.

Oyanedel, J., Alfaro, J., Varela, J. \& Torres, J. (2014). ¿Qué afecta el bienestar subjetivo y la calidad de vida de las niñas y niños chilenos? Resultados de la Encuesta Internacional sobre Bienestar Subjetivo Infantil. Santiago de Chile: LOM.

Parfenoff, S. \& Paul, J. (1989). Measuring daily stress in children. Poster presentado en la reunión anual del Society for Research in Child Development. Kansas City.

Putwain, D. (2007). Researching academic stress and anxiety in students: some methodological considerations. British Educational Research Journal, 33(2), 207-219.

Putwain, D. (2009). Assessment and examination stress in Key Stage 4. British Educational Research Journal, 35(3), 391-411. 
Quintard, B., Constant, A., Bouyssou-Gauthier, M.-L., Paul, C., Truchetet, F., Thomas, P. ... \& Taieb, A. (2011). Validation of a specific health-related quality of life instrument in a large cohort of patients with psoriasis: the QualiPso Questionnaire. Acta Dermato-Venereologica, 91(6), 660-665.

Seiffge-Krenke, I. (2000). Causal links between stressful events, coping style, and adolescent symptomatology. Journal of Adolescence, 23(6), 675-691.

Sun, J., Dunne, M. P., Hou, X. \& Xu, A. (2011). Educational Stress Scale for Adolescents: Development, Validity, and Reliability With Chinese Students. Journal of Psychoeducational Assessment, 29(6), 534-546.

Sun, J., Dunne, M., Hou, X. \& Xu, A. (2013). Educational Stress among Chinese Adolescents: Individual, Family, School and Peer Influences. Educational Review, 65(3), 284-302.

Trianes, V., Blanca, M., Fernández, F., Escobar, M., Maldonado, E. \& Muñoz, Á. (2009). Evaluación del estrés infantil: Inventario Infantil de Estresores Cotidianos ( IIEC ). Psicothema, 21, 598-603.

Vázquez, C., Crespo, M. \& Ring, J. (2000). Estrategias de afrontamiento. En A. Bulbena, G. Berrios \& P. Fernández de Larrinoa, (Eds.), Medición clínica en Psiquiatría y Psicologia (pp. 425-446). Barcelona: Masson.

Wells, N. M. \& Evans, G. W. (2003). Nearby Nature: A Buffer of Life Stress among Rural Children. Environment \& Behavior, 35(3), 311-330.

Recibido: 10 de noviembre, 2014 Aceptado: 16 de enero, 2015 